usual manner. The loose pieces of bone came away, and there was left a semicircular opening on it:; left side, which measured three inches by two inches and a half. The line of fracture extended forwards and around the skull to the other side of the head. The middle fossa of the base of the skull was broken into sercral pieces, and the roof of the orbit was slightly split. These bones were firmly fixed, and were united by bone. The fracture extended forwards across the frontal bone just below its eminences, and thence downwards to the lambiloidal suture, where it terminated. Wherever fracture had occurred, active repair was in progress; and this was particularly the case with that on the right side of the skull. Here the broken edges of bone had become firmly united, and the interposed connecting substance was hard kony tissue.

Considerable inflammatory action had been developed around all the edges of the broken bone, and for an inch or more on either side of them. They were also nicely rounded. The internal surface of the skull was covered with minute bony granulations, producing an uneven rough surface. The bones were highly vascular.

It would now appear, some months after the maceration and drying of the calvaria, that the aperture left after the removal of the comminuted bone has contracted, for the pieces of bone removed immediately after the injury will not fit into the hole. This contraction may have been the result of some action during life, or of contraction by the process of drying.

REntaks. In this case the three periods are well marked. The first extends from the receipt of the injury to the evenin of the seventh day, when marked indications of ararhnitis existed. I happened to be at the Hospital when the boy was brought there, and therefore no time was lost in removing the depressed bone-a practice now, I believe, aclinowledgerl to be the correct onc in all cases of compound comminuted fracture of the skull with depression. lieaction came on very slowly, for not until the second day was it fully established. This might perhaps be explained by the severity and extent of the injury. The amount of injury to the frontal bone detected after death may explain the extensive ecchymosis of the forehead, eyelids, and conjunctiva. The hammer had evidently acted as a wedge, and had lifted the calvaria, as it were, from the base of the skull.

During this time the patient was kept perfectly quiet ; milk diet was given; the head shaved; evaporating lotion applied over it; and alout five grains of calomel were administered, in divided doses.

The second period was very characteristic, faintly indicated in the morning by the pulse becoming faster and more sharp ; convulsive movenents of the right facial muscles: towards evening, well marked symptoms of arachnitis existed; pyrexia ; pulse hard, small, 90 per minute; the muscles of the right arm paralytic. Venesection was performed, and its influence was most striking. The pulse became full, less rapid, and quite compressible. Every indication of arachnitis subsided, and he seemed to be progressing favourably towards recovery until the thirty-fourth day after the injury, when he vomited, and slight pyrexia existed.

The third period now commences, when, I presume, inflammation of an inactive kind occurred in the left hemisphere, and the cyst containing the pus began to form. The very thick walls which this cyst exhibits would lead one to conjecture that a considerable period of time would be occupied in its formation; and I have thus assigned forty-two days for its development. During this time there existed, at intervals, abundant indications of great cerebral disturbance, but never of such a character, or sufficiently diagnostic of the formation of pus, or, even if there was a probability of its existence, of its precise locality. The possibility of suppuration existing frequently crossed my mind; and, had there been any facts to justify a puncture of the brain, I might have been induced to do so.

The necropsy, however, proved that we should not have reached the abscess in the brain without inflicting great injury upon that important organ; and a fatal result might then perhaps have occurred, even sooner than it did.

The Aberdeen University. The Earl of Stanhope has accepted the Lord Rectorship of the Aberdeen University in terms of a vote giving him a majority in all the nations, and promises to deliver an inaugural address. The town council are likely to confer on his lordship the freedom of the city.

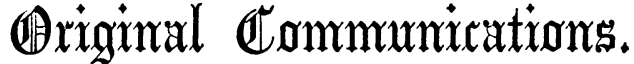

\section{CASE OF HEPATIC ABSCESS, SUDDENLY FATAL.}

By Sajict. Taylor Chadwick, M.D.Edinb., and M.R.C.S. England.

JAmes MLAsox, aged 17 , an athletic youth, who had invariably enjoyed most excellent health, was on Monday, February $2: \mathrm{nd}$, 1858 , about eight o'clock in the evening, taken suddenly ill with severe pains about the lower part of the abdomen. At the time of the seizure he was executing some errands, at a distance of two miles from his residence.

On his arrival home, his father dispatched a messenger to my surgery, requesting me to send something to afford him relief, as his sufferings became more and more aggravated. I furnished for him two pills, each containing one grain of calomel, with half a grain of opium; also a mixture of castor oil, compound tincture of rhubarb, and mucilage, and directed that one of the pills, with half of the mixture, should be administered immediately, and the remaining pill and mixture in three hours if relief were not obtained. The abdomen was to be assiduously fomented, and bran poultices were ordered to be applied. Early on the following morning I was informed that the remedies had afforded him considerable benefit; still his father was anxious that I should visit him in the course of the day. But as he was taken suddenly worse soon after the person had left home, a second messenger was also sent to urge my immediate attendance.

On my arrival at the house, which was soon after ten o'clock in the morning, $I$ found the patient in bed, complaining of pain over the whole course of the abdomen, of a very severe character. The features were pinched; the countenance exceedingly anxious; the breathing heavy and laborious: there was no pulse at the wrist; the skin was dry and moderately warm; the tongue moist, and slightly furred; the abdomen swollen and tense. The head was free from pain; there had been no wandering; I found him perfectly conscious, able to reply to any question clearly and distinctly.

He assured me that up to the period of the present attacic he felt quite well and vigorous, and had that day attended to his usual avocation, which was that of an agriculturist, his fathe being an extensive farmer at Heaton in this neighbourhood.

$\mathrm{He}$ further stated, in answer to my inquiries, that he hail not passed any urine since noon the day preceding, nor had he experienced any inclination to do so ; that his bowels had acted shortly after the invasion of the pain. He had been sick three or four times during the night, but had vomited very little.

When they arrived with him at home he earnestly entreated them to allow him to be placed on a sofa in the sitting-room, on which he remained during the night, and until about nine o'clock the following morning, when he was prevailed upon to go up stairs to bed. The effort of changing his room hurried him greatly, although he had been carefully assisted in doin so; and it was from this moment that he became rapidly worse; the breathing being more embarrassed, and the pain greatly increased.

I called his parents aside, and intimated to them my conviction he could not survive over an hour. I remained with him up to the time of his death, which occurred in about forty minutes after my arrival at the house. He continued perfectly sensible to the last.

In consequence of the distension of the abdomen, and no urine having been passed for twenty-two hours, I introduced a catheter; no urine, however, escaped. I also gave him stimulants, and directed a sinapism to be applied to the scrobiculus cordis; the weight even of that was more than he could conveniently bear. On the day succeeding his death I had an in terview with the father, and urged him to allow a post mortem examination to be made.

It was not without considerable difficulty that I succeeded in procuring his consent, which was granted with the positive as surance that the inspection should not be extended further than the cavity of the abdomen. At the hour fixed for the examination I was obliged to go into the country on a case of emergency. Dr. Settle, however, who conducted the examinstion, was kind enough to accompany my assistant; and I am indebted to him for the subjoined report of the post mortem appearances. 
INSPECTION twenty-four hours after death. The general appearance of the body was that of a stout, well-grown young man, of about 17 or 18 vears of age. The abdomen was very much distended, and, or an incision being made, gave exit to a large quantity of gas. There were found three to four quarts of fluid in the peritoneum, the first portion removed being clear; that from the pelvis and dependent parts resembled a mixture of pus and serum. The general vascularity of the peritoneum was slightly increased; all the abdominal viscera appeared healthy with the exception of the liver, on examining which there was found a large opening, commencing at its anterior edge, where the suspensory ligament becomes attached to it, and extending into the substance of the right lobe, forming a very extensive cavity, from which the pus found in the abdomen had evidently escaped. Pressure caused an alditional flow of pus. 'The gall-bladder was distended with bile. The bladder contained no urine.

RFmarks. From the symptoms, attack, and mode of death, I was strongly impressed with the idea that there must have been rupture, or perforation of some viscus.

The fact of the patient not having passed urine for almost an entire day, viz., twenty-two hours, coupled with that of the bladder being found perfectly empty on the introduction of the catheter, led me to think it possible that the bladder was ruptured, and its contents extravasated into the peritoneum. On the other hand, the absolute denial of his having sustained any external violence militated against such a supposition. Again, it was difficult to reconcile it as being a case of perforation of the intestine, inasmuch as there had been neither diar. rhea nor dysentery to lead on to ulceration or perforation.

It must be conceded that the case is one not devoid of in terest, when we reflect that an abscess should go on to formation without any manifestations or derangement of the general health. Moreover, the suppression of the renal secretion is worthy of observation.

\section{ON DISEASES OF JOINTS.}

By Holmes Coote, Esq., F.R.C.S., Assistant-Surgeon to St. Bartholomew's Hospital, and to the Royal Orthopædic Hospital, etc.

\section{IATTERAL CURVATURE OF THE SPINE.}

$$
\text { [Concluded from page 188.] }
$$

THE practice of keeping patients afflicted with lateral curvature of the spine, for months, and even years, upon reclining boards, is in every way reprehensible. I doubt whether existence under such circumstances would be acceptable to a person with healthy mind. To those subject to perverted feeling and hysterical emotions such a plan would obviously encourage the morbid tendency; and there are few surgeons who cannot recal to mind cases in which, to use the words of Dr. Prout, "the ruling passion is displayed in attempting to excite pity and commiseration, where the erotic sentiment cannot be excited."

Now, some practitioners keep their patients on the back; others on the face; others on the side. Of these, the first is the most reasonable as affording rest. The other two must be inconvenient and disagreeable to a degree. I grant that there are cases in which the deviation from the straight line disappears where the patient assumes the recumbent posture; but what will ensue from the continuance of such a practice? Judging from what we witness in bed-ridden cases, I should expect the ligaments first to lose their tone, and then to contract and form morbid adhesions. As regards the bones, they lose their earthy material, and become light and spongy. $\dot{A}$ vertebral column of usual size taken from an adult and dried, weighed one pound seven ounces and a half ; one taken from a person long confined to bed, weighed, under similar circumstances, only thirteen ounces and a half.

This change pervades the whole osseous system; the cancellous texture of the long bones disappears, and a shell filled with brownish fat remains. This is called excentric atrophy and $I$ have known it proceed to such an extent that the femur has broken in no violent handling; or has been so light that the saw could scarceiy be used in amputation. What must ensue when the patient rises after a long period of this restraint? Can we expect healthy tone in any of the normal structures? On the contrary, the patient is in that condition most prone, from general debility, to physical deformity.

There are cases of incipient lateral curvature in young per. 205 sons (of the class " general vielding of the vertebral column") which may be treated without instrumental supports. Abstinence from employments which call forth the exercise of one side of the body in preference to the other; fresh air; out-door occupations; and rest whenever a feeling of fatigue is experienced, may, in conjunction with the bodily changes effected by growth, succeed in arresting, if not in entirely removing, the deformity. But the patient requires watching, for the tendency to disease continues, and it may become progressive long after puberty.

CASE I. John B-, aged 31 , a well built, yet slightly made man, of pale complexion, waiter at an hotel, applied at the Royal Orthopredic Hospital, February 25th, 1858, complaining of weakness in the loins, loss of power in the lower extremities, fatigue after walking a mile or so, and "dropping" of the right shoulder upon rising of a morning. Upon examination, there was found general looseness of the vertebre, the spinal column yielding readily to the weight of the head and upper extremities. There was a slight double lateral curvature. In the recumbent, posture, the deviation very much disappeared. It was obvious that this man's employment was beyond his strength, and he was directed to wear the pelvic band with two crutches extending to the axillæ.

But whenever the curvature is established, the only method of affording relief is by the application of some constantly acting support, which will overcome the resistance of the contracted ligaments, and allow the elongated ligaments of the convexity to resume their normal proportions. These conditions are, in fact, fulfilled by the well known instrument in. vented by M. Tavernier, and called the lever-belt. Yet there is felt, by those who have used it, a want of power to act with accuracy against particular parts of the curve.

This deficiency is supplied by Mr. Tamplin's instrument, one equally well known, and acting by means of pads pressing in the situation required, by steel rods moved by cogwheels, and attached to a pelvic band. The apparatus has been modified according to the fancies of other practitioners, some adding a screw here or there; the principle, however, remains precisely the same as laid down by Mr. Tamplin, and of its utility no better proof can be afforded than the acquiescence in its superiority by the late Mr. Lonsdale some short time before his death. Those desirous of learning more about the instrument may either consult the work upon the subject (On Lateral Curvature of the Spine, by R. W. Tamplin. London, 1852), or see it any time at most surgical instrument makers, such as Mr. Ferguson, of Giltspur Street.

It is not sufficient to direct the patient to put on the instrument, and then leave the further progress of the case to chance. Most careful watching is necessary, and the extending force must be used slowly, with patience and skill. If there is only one long curve, generally directed to the right, a single pad will be sufticient to act upon it. But if there be a double curve, counter pressure by a second pad, also moveable, must be likewise exerted. The steel rods, which support the pads, must move not only from side to side, but from before backwards, that they may accurately adapt themselves to the figure, and keep up pressure whatever amount of change may ensue. Finally, the crutches under the axilla, extending from the pelvic band, require movement in every direction, that proper support may be given without fretting or excoriation. The crutch on the concavity should rise well into the axilla, and be sup. plied with means of being elongated; for the body of a person suffering from lateral curvature may be raised from half an inch to two inches, or even more, when corresponding instrumental changes, without removal of the apparatus, become requisite.

The surgeon should himself turn the screw to which the compressing pad is affixed on alternate days, or at least twice a week, when it will be noticed that, as the spinal deformity is relieved, constitutional symptoms under which the patient has long suffered vanish accordingly. A lady, at present under my care, has for many years suffered from violent periodical headaches, which have almost entirely disappeared sirice the deformity of the vertebral column has been relieved. A second patient, whose case is recorded in this Joursal (p. 144), experienced such irregularities in the action of the heart, that she feared to go out alone lest she should faint in the streets. The replacement of the viscera of the thorax, consequent upon the correction of the spinal deformity, removed this unpleasant sensation. A third patient was relieved by similar treatment of distension of the intestine by flatus and general inactivity of the alimentary canal, alternating with occasional attacks of spasm, tenesmus, and diarrhwea. A fourth patient (whose case is related at p. 187 of this Jounsar), was relieved of a set of 